\title{
Special MISO-SAR and MIMO-SAR Modes for Bidirectional Imaging
}

\author{
Hai Jiang, ${ }^{1,2}$ Hongjun Song, ${ }^{1}$ Lei Guo, ${ }^{1,2}$ and Wei Wang ${ }^{1,2}$ \\ ${ }^{1}$ Institute of Electronics, Chinese Academy of Sciences, Beijing 100190, China \\ ${ }^{2}$ University of Chinese Academy of Sciences, Beijing 100190, China \\ Correspondence should be addressed to Hai Jiang; bradley0226@163.com
}

Received 15 January 2014; Accepted 27 February 2014; Published 31 March 2014

Academic Editor: Wei Xu

Copyright (c) 2014 Hai Jiang et al. This is an open access article distributed under the Creative Commons Attribution License, which permits unrestricted use, distribution, and reproduction in any medium, provided the original work is properly cited.

\begin{abstract}
The paper proposes special multiple-input single-output synthetic aperture radar (MISO-SAR) and multiple-input multiple-output SAR (MIMO-SAR) for bidirectional imaging, which can simultaneously illuminate two areas from different directions in azimuth. For the proposed MISO-SAR, two subpulses with the same carrier frequency and phase coding are transmitted with different azimuth directions by switching the phase coefficients in the transmit modules, and echoes corresponding to the subpulses are received by the main lobe and the first grating lobe of the whole antenna. To suppress mutual interference, the two subpulses are transmitted with different range-frequency bands, and their echoes are demodulated and recorded in different channels in the proposed MIMO-SAR. This paper presents the system design of these modes and analyzes their azimuth ambiguity to signal ratio (AASR). Besides, simulation results on points are carried out to validate the proposed bidirectional imaging modes.
\end{abstract}

\section{Introduction}

The repeated acquisition of synthetic aperture radar (SAR) images is very useful for multiple observation applications, such as moving targets detection, terrain change detection, and velocity measurements by along-track interferometry [13]. For a SAR satellite, the time lag between two acquisitions must be more than several hours. However, for iceberg drifts or ship velocity measurements $[4,5]$, the time lag should be less than several minutes. The single channel bidirectional SAR imaging mode is proposed in [1] and implemented via a phased planner antenna to generate both main lobe and grating lobes to illuminate different areas. This imaging mode was first achieved by TerraSAR-X satellite [6-10]. TerraSAR-X and TanDEM-X have several new modes and finished some commissions in [7, 11-14]. Another advantage for bidirectional SAR mode is that the simultaneous imaging into two directions can obtain two widely separated Doppler subbands, which can offer many different interferometry or GMTI applications [15-19].

The major drawback of the single channel bidirectional SAR imaging mode is the high azimuth ambiguity to signal ratio (AASR) level caused by the grating lobes. To suppress the high AASR level, a pulse repetition frequency (PRF) value higher than the one in the conventional stripmap mode with the same antenna length is required or the azimuth processed bandwidth is reduced. Therefore, the bidirectional imaging mode is balanced by the limited swath width due to the higher PRF and/or by the impaired azimuth resolution due to the reduced azimuth processed bandwidth.

In this paper, two special imaging modes for bidirectional imaging based on the phased planner antenna are proposed. In the first mode, two subpulses are transmitted in turn with different azimuth antenna beams by switching the phase coefficients in the transmit modules, and echoes of the two subpulses are simultaneously received by the main lobe and the first grating lobe of the whole antenna and recorded in a single channel. This imaging scheme with two transmitted subpulses and a single receive channel is named as multipleinput single-output SAR (MISO-SAR) for bidirectional imaging. To further distinguish the echoes, two subpulses are transmitted with different frequency bands, and then their echoes are demodulated and recorded in two channels. As 


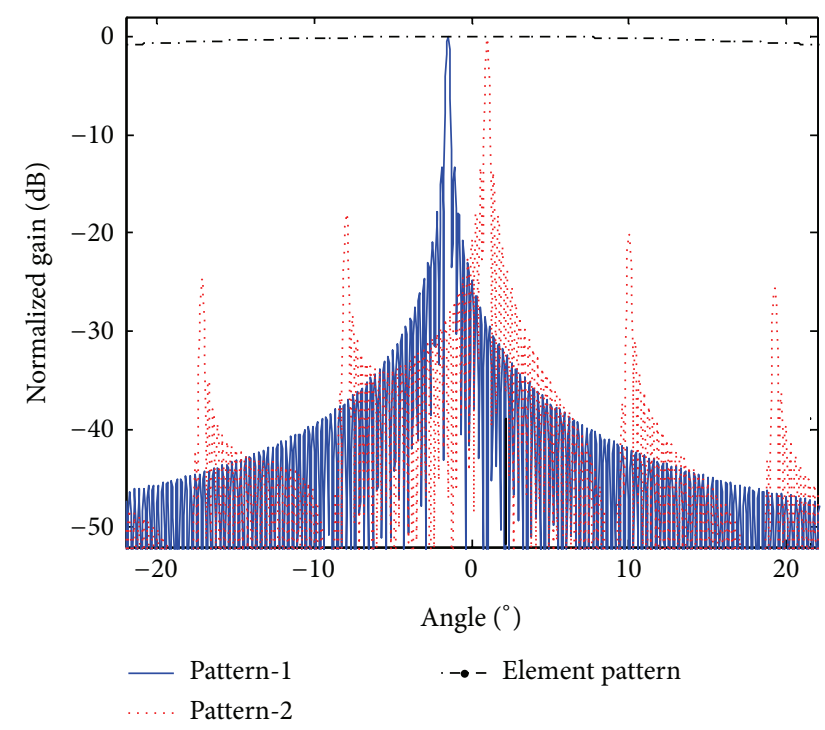

FIGURE 1: Antenna patterns of the phased array antenna.

a result, the second imaging mode is named as multipleinput multiple-output SAR (MIMO-SAR) for bidirectional imaging. Compared with the bidirectional imaging mode in [1], the two proposed imaging modes are with the lower AASR level. The system design and signal processing of the proposed modes are given in detail. Furthermore, simulation experiments on point targets are carried out to validate the proposed bidirectional imaging modes.

The paper has five sections. Section 2 focuses on presenting two proposed imaging modes for bidirectional imaging. The system design and AASR analysis of the two modes are given in Section 3. The imaging approach of two modes and simulation experiments on point targets are presented in Section 4. The paper is concluded in Section 5.

\section{Special MISO-SAR and MIMO-SAR Modes}

2.1. Antenna Pattern of the Phased Array Antenna. To implement antenna beam scanning in both azimuth and elevation, a phased two-dimension (2D) planar antenna is usually adopted in the future spaceborne SAR missions. According to the working principle of the phased array antenna, the one-way antenna pattern of the phased array antenna can be written as follows [4]:

$$
G(\theta)=G_{e}(\theta) \cdot\left|\frac{1}{K} \sum_{k=0}^{K-1} C_{k, T} \cdot \exp \left(j \frac{2 \pi k}{\lambda} L_{\mathrm{ae}} \sin \theta\right)\right|,
$$

with

$$
G_{e}(\theta)=G_{0} \cdot\left|\sin c\left(\frac{L_{\mathrm{ae}}}{\lambda} \sin \theta\right)\right|,
$$

where $G_{e}(\theta)$ indicates the antenna pattern of the element antenna, $K$ is the number of the transmit/receive (T/R) modules, $\lambda$ is the wavelength, and $L_{\mathrm{ae}}$ is the length of the element antenna. To avoid the grating lobes during antenna beam steering, the length $L_{\mathrm{ae}}$ should be

$$
L_{\mathrm{ae}} \leq \frac{\lambda}{1+\left|\sin \theta_{s, \max }\right|},
$$

where $\theta_{s, \max }$ is the maximal steering angle. To implement the desired antenna beam pointing direction, the phase coefficients of the $T / R$ modules are expressed as

$$
\begin{gathered}
C_{k, T}=a_{k, T} \cdot \exp \left(j \frac{2 \pi k}{\lambda} L_{\mathrm{ae}} \sin \theta_{s}\right), \\
C_{k, T, g}=a_{k, T} \cdot \exp \left(j \frac{2 \pi}{\lambda} \cdot\left\lfloor\frac{k}{M}\right\rfloor \cdot L_{\mathrm{ae}} \sin \theta_{s}\right),
\end{gathered}
$$

where $a_{k, T}$ is constant, $\theta_{s}$ indicates the desired antenna beam pointing direction, and $M$ is the number of the T/R modules with the same phase coefficient. With the phase coefficient of (2), the distance between the main lobe and the first grating lobe is

$$
\Delta \theta=\frac{\lambda}{M \cdot L_{\mathrm{ae}}} .
$$

Figure 1 shows antenna patterns of the phased array antenna with phase coefficient coding of (4) and (5), where the element antenna $L_{\mathrm{ae}}$ is $0.02 \mathrm{~m}$, the wavelength is $0.03125 \mathrm{~m}$, the number of elements is 320 , and $M$ is 20 . Furthermore, the antenna beam pointing direction $\theta_{s}$ in (4) is $-1.5^{\circ}$, while $\theta_{s}$ in (5) is $1^{\circ}$.

2.2. Acquisition Geometry. Figure 2 shows the proposed modified bidirectional SAR acquisition geometry with simultaneous fore and aft acquisitions. In the bidirectional SAR imaging mode in [1], each pulse is transmitted to two different areas in azimuth and its corresponding echoes are received by the main lobe and the first grating lobe of the same azimuth antenna pattern. However, the large transmitted radar pulse is divided into two subpulses to be transmitted into different azimuth areas in the proposed modified bidirectional SAR, and the subpulses are transmitted by different azimuth antenna patterns by steering the azimuth antenna beam as shown in Figure 2(a). The echoes of two subpulses from two azimuth areas are received by the main lobe and the first grating lobe of the whole azimuth antenna, respectively.

In the first case, two subpulses are with the same carrier frequency and phase coding, while their corresponding echoes are simultaneously received and sampled in a single channel. Therefore, this imaging scheme is named MISOSAR. To suppress the interference between echoes, two transmitted subpulses could be with different carrier frequencies, and their corresponding echoes are received and sampled with different channels. As a result, the second imaging scheme is named MIMO-SAR.

Figure 3 shows azimuth antenna patterns of the proposed modified bidirectional SAR acquisition. It demonstrates that the well-behavior transmitting antenna pattern may suppress the power of grating lobes and side lobes of the azimuth receiving antenna pattern. In Figure $3, M$ is 10 and the 


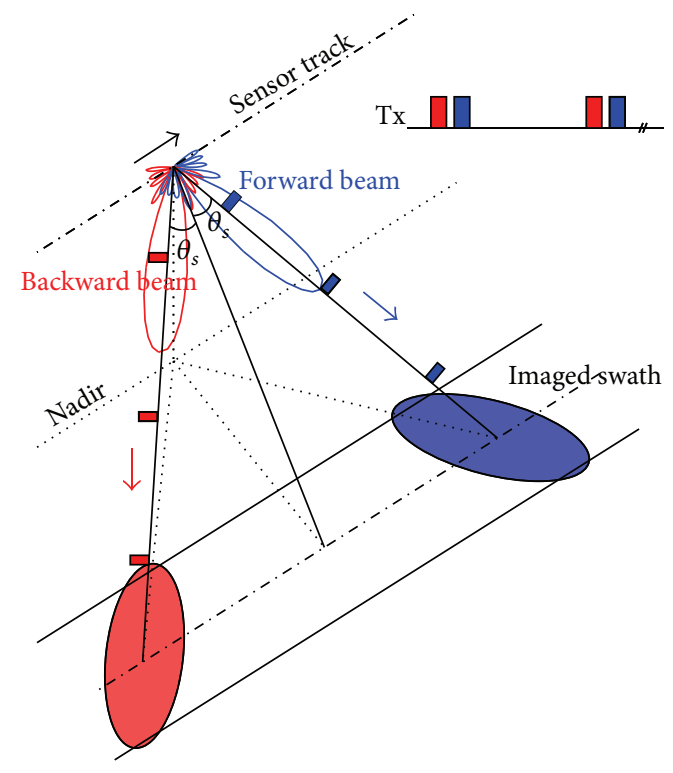

(a)

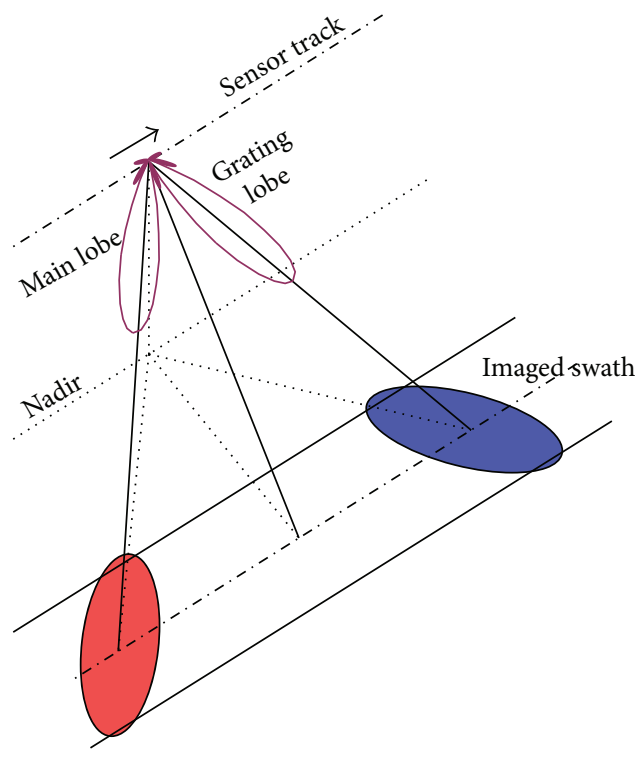

(b)

FIGURE 2: Modified bidirectional SAR acquisition geometry with simultaneous fore and aft acquisitions: (a) radar pulses transmitting and (b) radar echoes receiving.

angular interval between the main lobe and the first grating lobe is $8.952^{\circ}$. Therefore, the azimuth backward and forward beam pointing direction is $-4.476^{\circ}$ and $4.476^{\circ}$, respectively. Furthermore, the main lobe and the first grating lobe share the same antenna gain and are with $4 \mathrm{~dB}$ gain reduction compared with the main lobe of the azimuth transmitting beam pattern.

\section{AASR Analysis and System Design}

3.1. AASR Analysis. For the proposed MISO-SAR system, the AASR for the backward area and the forward area are computed as follows:

$$
\begin{array}{r}
\operatorname{AASR}_{b}=\left(\sum _ { k \neq 0 } \int _ { - B _ { a } / 2 + f _ { d c , b } } ^ { B _ { a } / 2 + f _ { d c , b } } \left[G_{t, b}\left(f_{a}+k \cdot \mathrm{PRF}\right)\right.\right. \\
\left.+G_{t, f}\left(f_{a}+k \cdot \mathrm{PRF}\right)\right] \\
\left.\cdot G_{r}\left(f_{a}+k \cdot \mathrm{PRF}\right) d f_{a}\right) \\
\times\left(\int_{-B_{a} / 2+f_{d c, b}}^{B_{a} / 2+f_{d c, b}}\left[G_{t, b}\left(f_{a}\right)+G_{t, f}\left(f_{a}\right)\right]\right. \\
\left.\cdot G_{r}\left(f_{a}\right) d f_{a}\right)^{-1},
\end{array}
$$

$$
\begin{array}{r}
\operatorname{AASR}_{f}=\left(\sum _ { k \neq 0 } \int _ { - B _ { a } / 2 + f _ { d c , f } } ^ { B _ { a } / 2 + f _ { d c , f } } \left[G_{t, b}\left(f_{a}+k \cdot \mathrm{PRF}\right)\right.\right. \\
\left.+G_{t, f}\left(f_{a}+k \cdot \mathrm{PRF}\right)\right] \\
\left.\cdot G_{r}\left(f_{a}+k \cdot \mathrm{PRF}\right) d f_{a}\right) \\
\times\left(\int_{-B_{a} / 2+f_{d c, f}}^{B_{a} / 2+f_{d c, f}}\left[G_{t, b}\left(f_{a}\right)+G_{t, f}\left(f_{a}\right)\right]\right. \\
\left.\cdot G_{r}\left(f_{a}\right) d f_{a}\right)^{-1}
\end{array}
$$

where $f_{a}=2 v \sin \theta / \lambda$ is the Doppler frequency, $v$ is the speed of the radar, $\theta$ is the squint angle, $B_{a}$ is the processed Doppler bandwidth, $G_{r}$ is the azimuth receiving antenna pattern, $k$ is an integer, $G_{t, b}$ and $G_{t, f}$ indicate the azimuth transmitting antenna patterns for the aft and fore directions, respectively, and $f_{d c, b}$ and $f_{d c, f}$ are the Doppler centroids for backward and forward imaging. For well-symmetric fore and aft azimuth directions, the AASR is connected to the spectral separation as the grating lobe is the strongest contribution of the ambiguous signal energy to the main lobe as shown in Figure 3.

Similar to the single channel bidirectional SAR system, the cyclic behavior of divergent and coincident folding also becomes visible by plotting the AASR versus the selected PRF as shown in Figure 4. The simulation parameters are listed in Table 1 . As the grating lobe moves relative to the main lobe, 


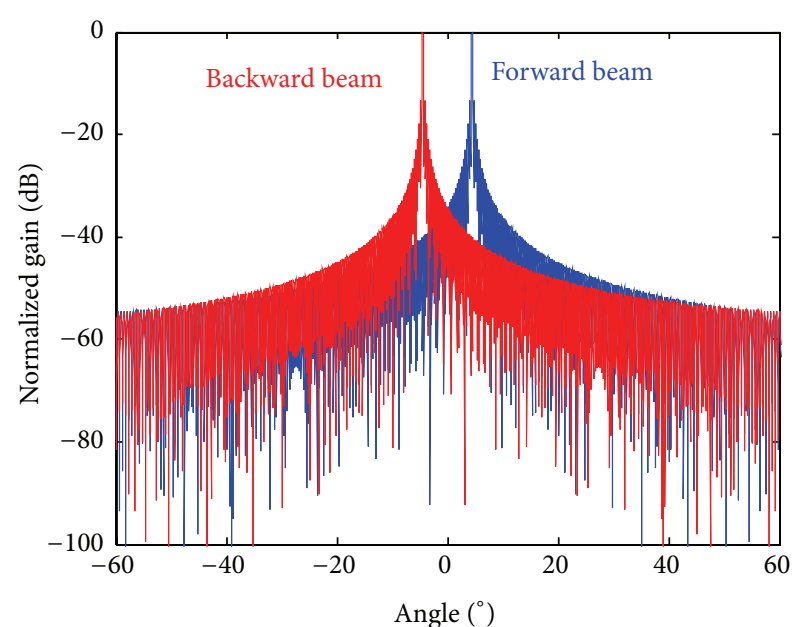

(a)

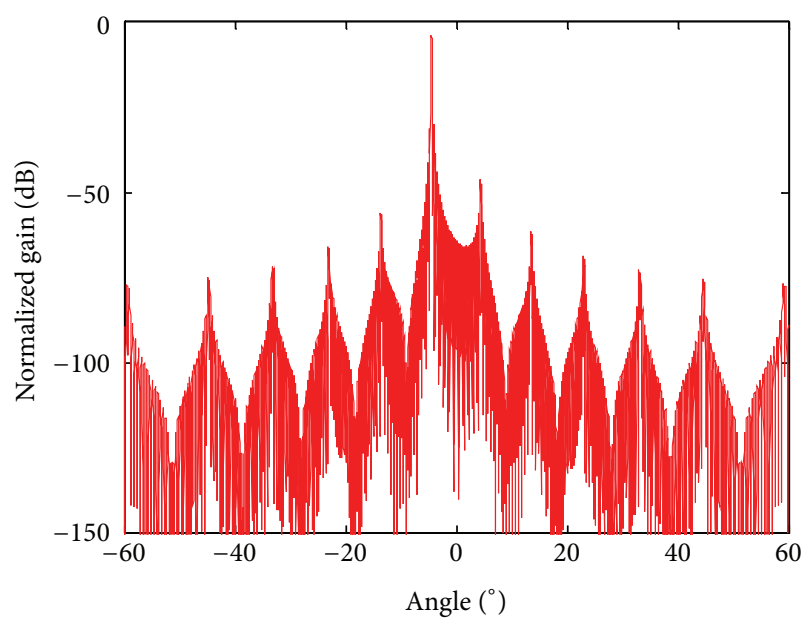

(c)

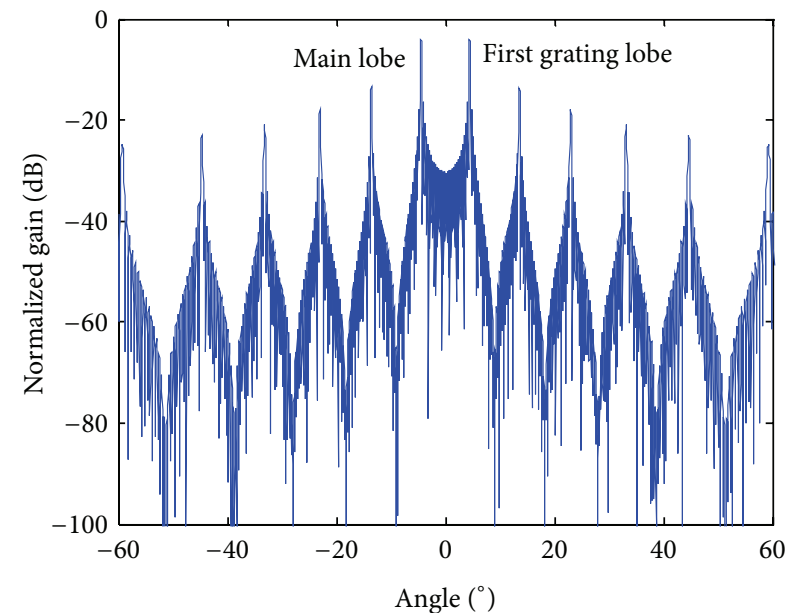

(b)

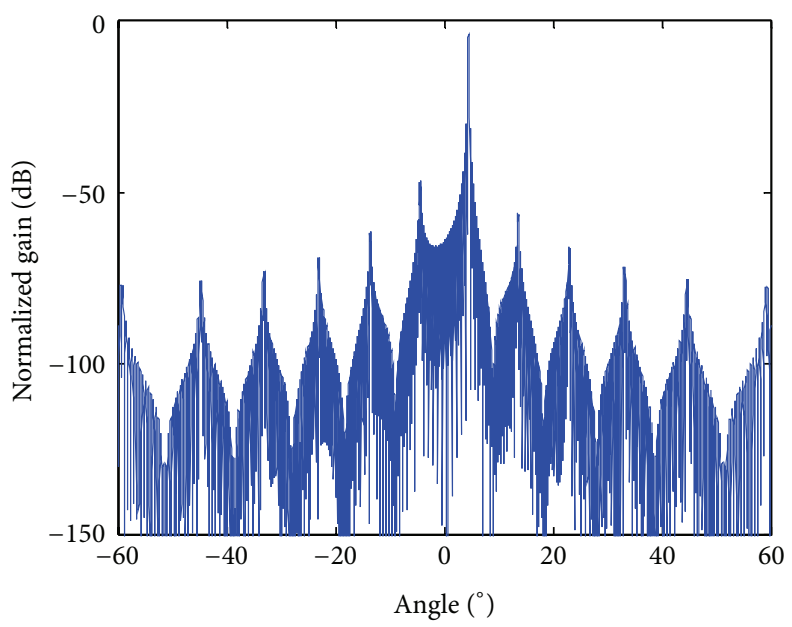

(d)

FIgURE 3: Azimuth antenna patterns of the phased antenna. (a) Transmitting antenna pattern. (b) Receiving antenna pattern. (c) Two-way antenna pattern for backward imaging. (d) Two-way antenna pattern for forward imaging.

TABLE 1: Simulation parameters.

\begin{tabular}{ll}
\hline Parameters & Value \\
\hline Antennal length $(\mathrm{m})$ & 6.4 \\
Azimuth element antenna length $(\mathrm{m})$ & 0.02 \\
Number of T/R in azimuth & 320 \\
Number of T/R modules with the same phase on receive & 20 \\
Carrier frequency in MISO-SAR $(\mathrm{GHz})$ & 9.6 \\
Carrier frequencies in MIMO-SAR $(\mathrm{GHz})$ & $9.4,9.8$ \\
Transmitted pulse duration $(\mu \mathrm{s})$ & 10 \\
Pulse bandwidth $(\mathrm{MHz})$ & 150 \\
Sampled frequency $(\mathrm{MHz})$ & 180 \\
Sensor velocity $(\mathrm{m} / \mathrm{s})$ & 7500 \\
Slant range $(\mathrm{km})$ & 700 \\
Squint angles for bidirectional imaging $\left(^{\circ}\right)$ & \pm 4.476 \\
\hline
\end{tabular}

the AASR oscillates between high and low values. The high value of the AASR is about $0 \mathrm{~dB}$ and keeps constant, since it reflects the coincident folding in the two-way antenna pattern for backward imaging and forward imaging. The low value becomes lower with increasing PRF, since Doppler spectra of backward imaging and forward imaging areas can be better separated by band-pass Doppler filtering. As the gain of the main lobe and the first grating lobe is the same in Figure 3(b), the AASR in the aft and fore images are equivalent. Compared with the AASR of the single channel bidirectional SAR system in [1], the AASR of the proposed MISO-SAR system is better than the conventional bidirectional SAR system, since the power of the grating lobes and side lobes of the azimuth receiving antenna pattern is suppressed by the azimuth transmitting antenna pattern as shown in Figure 2. In other words, for the desired AASR level (e.g., about $-18 \mathrm{~dB}$ ) as shown in Figure 4, the selected PRF should be more than $6500 \mathrm{~Hz}$ in the single channel bidirectional SAR system, while the selected PRF only should be more than $5100 \mathrm{~Hz}$ in the proposed MISO-SAR system. In this simulation, the processed Doppler bandwidth is related to the $6 \mathrm{~dB}$ beam 


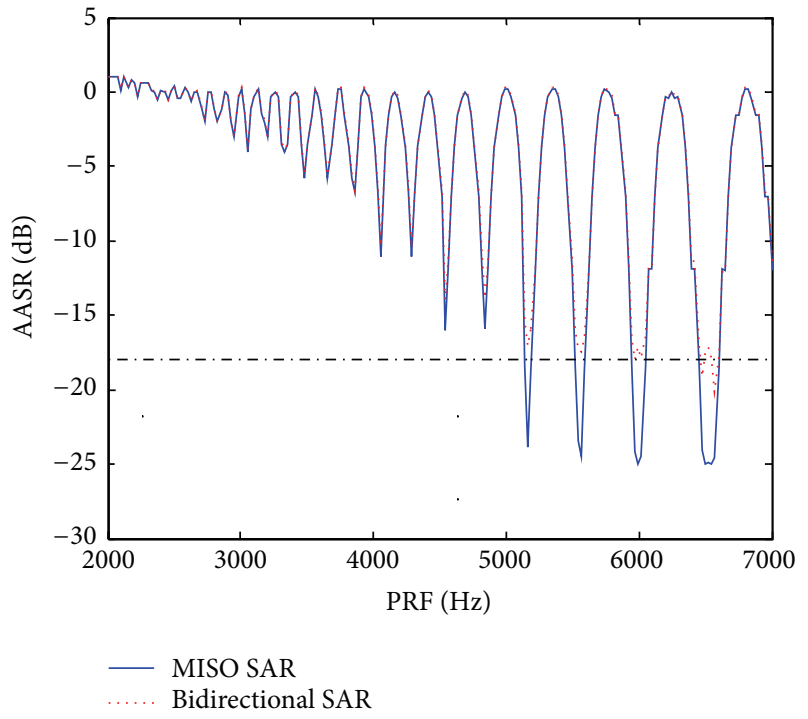

FIGURE 4: AASR of the proposed MISO-SAR and bidirectional SAR versus acquisition PRF.

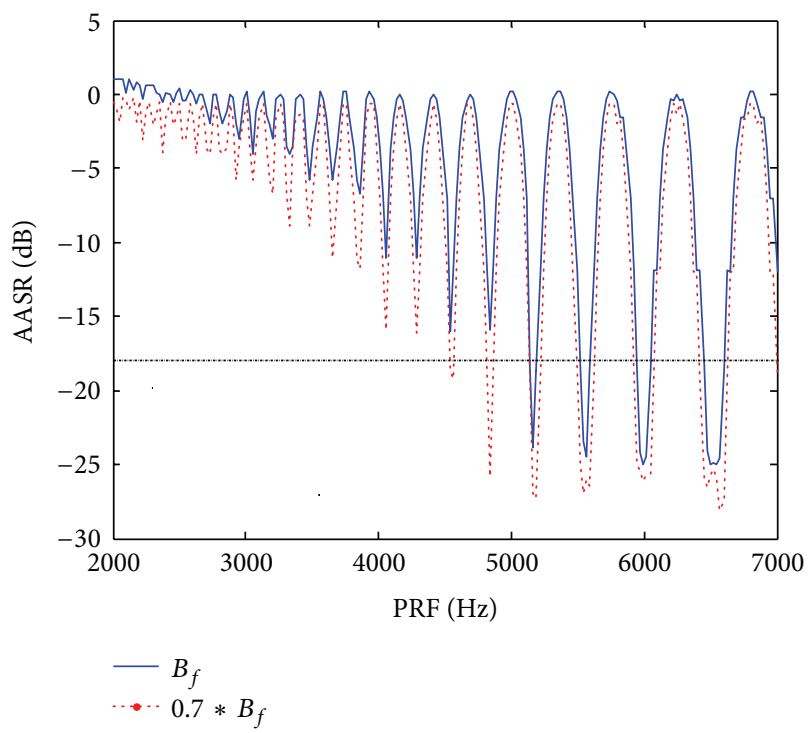

FIgURE 5: AASR of the proposed MISO-SAR versus acquisition PRF for different processed Doppler bandwidths.

width of the two-way azimuth antenna pattern and about $1454 \mathrm{~Hz}$.

Similar to conventional SAR imaging modes, the AASR level can be improved after reducing the processed Doppler bandwidth under the same condition as shown in Figure 5 but with the impaired azimuth resolution.

From (7), the high AASR level is caused by the interference between echoes of two transmitted subpulses in a single pulse repetition interval (PRI). To suppress the interference in the proposed MIMO-SAR for bidirectional imaging, two subpulses are transmitted with different rangefrequency bands and their echoes can be easily separated by range-frequency band-pass filtering. As two subpulses have

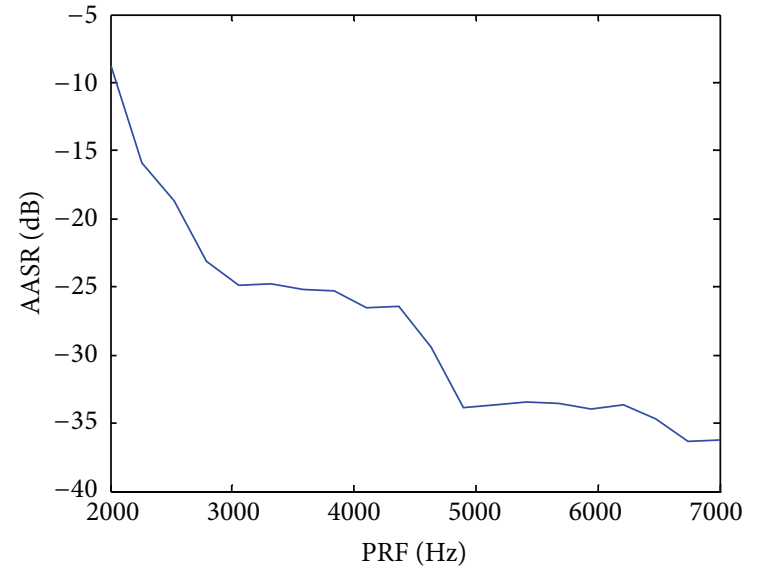

FIgURE 6: AASR of the proposed MIMO-SAR versus acquisition PRF.

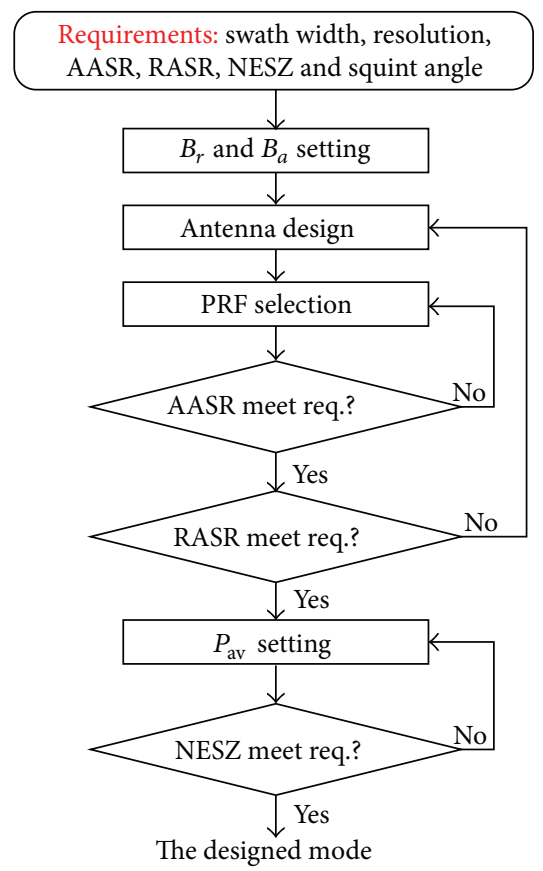

FIGURE 7: Block diagram of the system design of two proposed bidirectional SAR imaging modes.

different carrier frequencies, the AASR for the backward area and the forward area in the proposed MIMO-SAR system is computed as follows:

$$
\begin{aligned}
& \operatorname{AASR}_{b}=\left(\sum_{k \neq 0} \int_{-B_{a} / 2+f_{d c, b}}^{B_{a} / 2+f_{d c, b}} G_{t, b}\left(f_{a}+k \cdot \mathrm{PRF}\right)\right. \\
&\left.\cdot G_{r}\left(f_{a}+k \cdot \mathrm{PRF}\right) d f_{a}\right) \\
& \times\left(\int_{-B_{a} / 2+f_{d c, b}}^{B_{a} / 2+f_{d c, b}} G_{t, b}\left(f_{a}\right) \cdot G_{r}\left(f_{a}\right) d f_{a}\right)^{-1},
\end{aligned}
$$



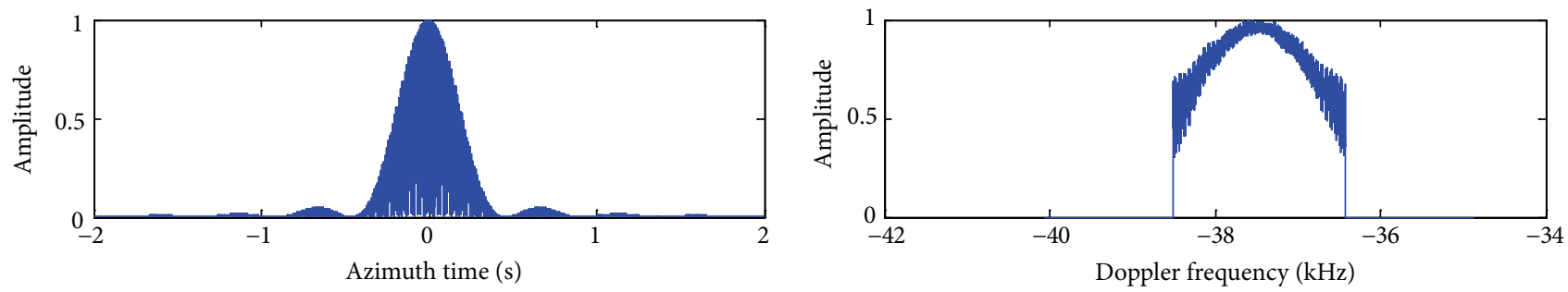

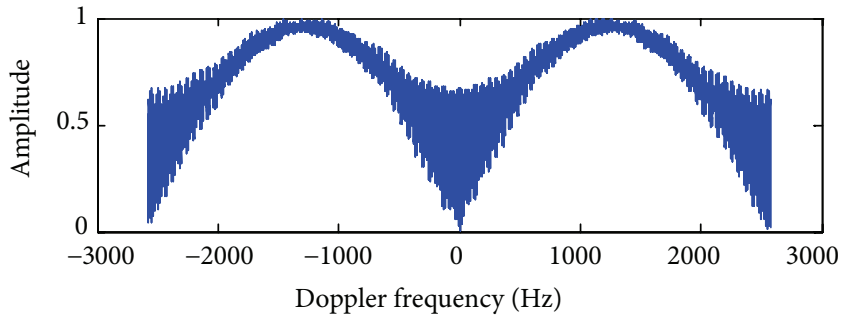

(a)

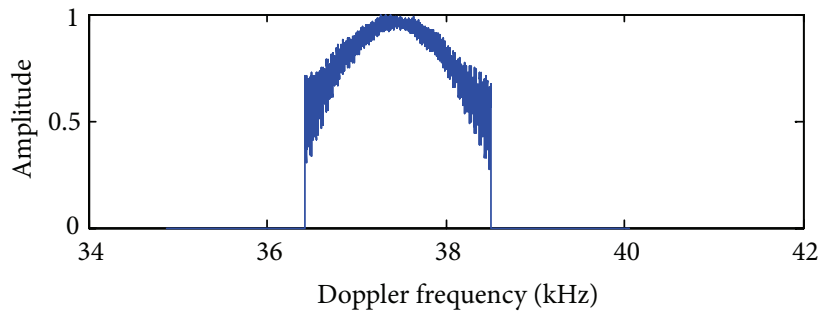

(b)
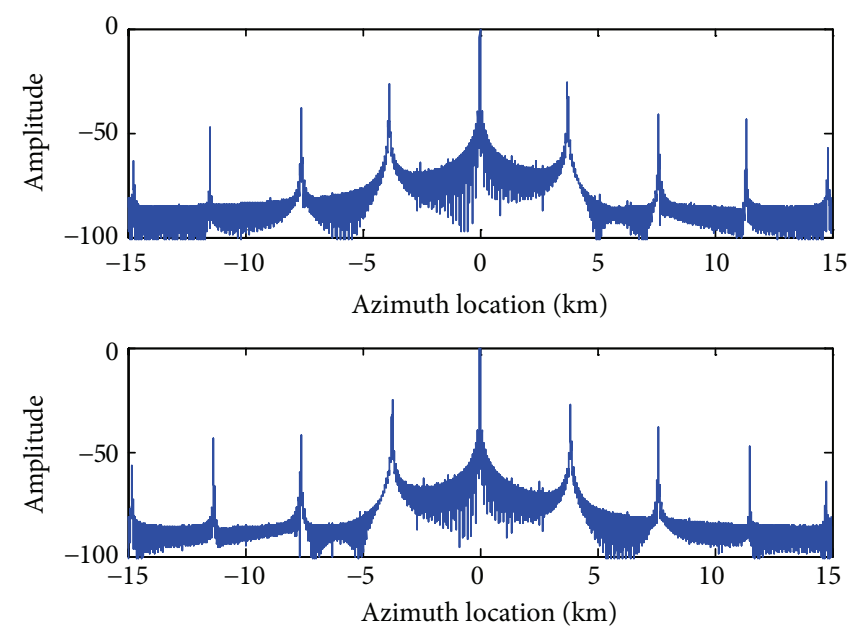

(c)

FIGURE 8: Simulation results of the proposed MISO-SAR with the PRF of $5170 \mathrm{~Hz}$. (a) Azimuth raw data in the time and Doppler frequency domains. (b) Doppler spectra after separation. (c) Imaging results from different azimuth directions.

$$
\begin{aligned}
& \operatorname{AASR}_{f}=\left(\sum_{k \neq 0} \int_{-B_{a} / 2+f_{d c, f}}^{B_{a} / 2+f_{d c, f}} G_{t, f}\left(f_{a}+k \cdot \mathrm{PRF}\right)\right. \\
&\left.\cdot G_{r}\left(f_{a}+k \cdot \mathrm{PRF}\right) d f_{a}\right) \\
& \times\left(\int_{-B_{a} / 2+f_{d c, f}}^{B_{a} / 2+f_{d c, f}} G_{t, f}\left(f_{a}\right) \cdot G_{r}\left(f_{a}\right) d f_{a}\right)^{-1}
\end{aligned}
$$

As a result, the AASR level of the proposed MIMOSAR system is much better than that of the MISO-SAR system. Figure 6 shows the AASR versus the selected PRF in the proposed MIMO-SAR system under the same condition.
3.2. The System Design. The block diagram in Figure 7 shows major system design steps of the two proposed bidirectional SAR imaging modes. The starting point is the desired system parameters of fore and aft images such as swath width, geometric resolution, AASR, range ambiguity to signal ratio (RASR), and noise equivalent sigma zero (NESZ), while fore and aft images are with the squint angles $\theta_{s}$ and $-\theta_{s}$, respectively. First, the transmitted pulse bandwidth $B_{r}$ and the processed Doppler bandwidth $B_{a}$ related to the azimuth antenna length are determined by the desired geometric ground resolution and azimuth resolution, respectively. According to the squint angle $\theta_{s}$ for bidirectional imaging, the number of $\mathrm{T} / \mathrm{R}$ modules and the azimuth beam steering raw controlled by the phase coefficients in (4) and (5) can be set. The PRF selection is looking for the satisfied AASR level. Furthermore, in addition to reducing the selected PRF, we can 

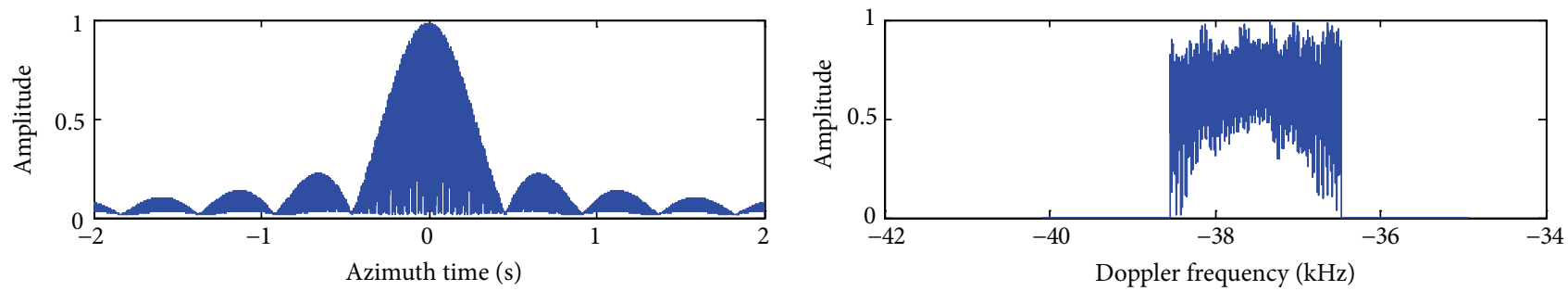

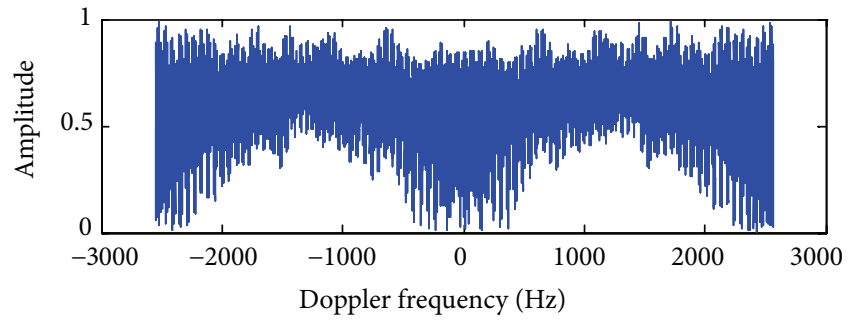

(a)

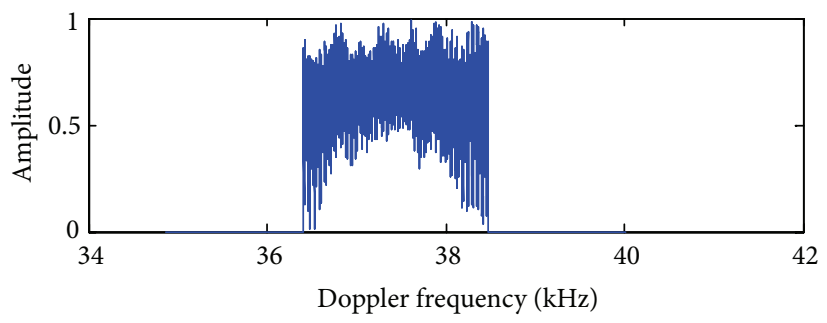

(b)
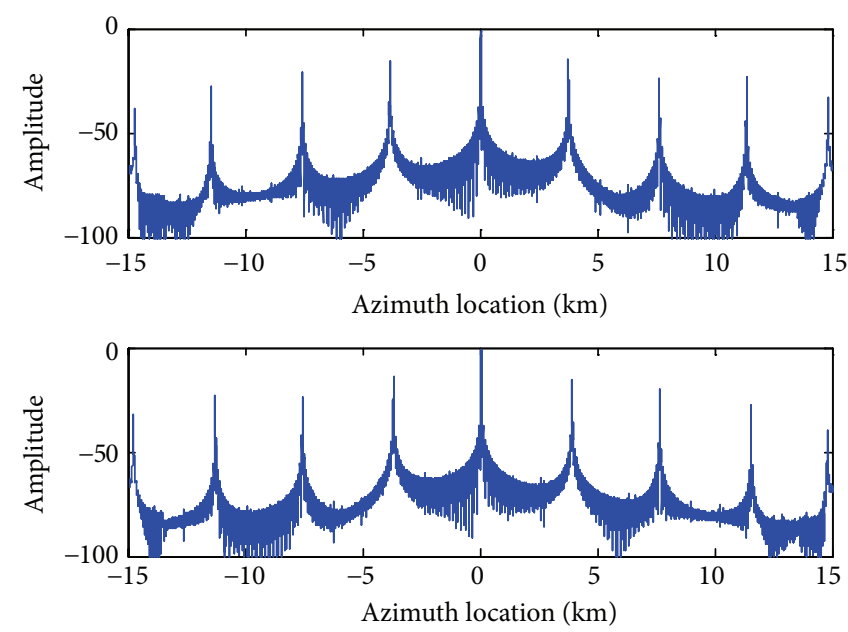

(c)

FIGURE 9: Simulation results of the original bidirectional SAR with the PRF of $5170 \mathrm{~Hz}$. (a) Azimuth raw data in the time and Doppler frequency domains. (b) Doppler spectra after separation. (c) Imaging results from different azimuth directions.

enlarge the antenna height to improve the RASR level. Finally, the average power $P_{\mathrm{av}}$ is set to obtain the desired NESZ.

The major difference between the proposed MISO-SAR and MIMO-SAR for bidirectional imaging is that two subpulses are transmitted with different range-frequency bands to avoid mutual interference. As a result, a lower selected PRF is required for the desired AASR level in the MIMOSAR system than in the MISO-SAR system under the same condition as shown in Figures 5 and 6. However, since the AASR oscillates between high and low values in the MISOSAR system, a small PRF range should be selected for a better AASR level.

\section{Raw Data Processing and Simulation}

To validate the proposed MISO-SAR and MIMO-SAR for bidirectional imaging, simulation experiments on point targets are carried out. Simulation parameters are listed in Table 1.

In the proposed MISO-SAR for bidirectional imaging, a much higher PRF than in the conventional stripmap case with the same antenna length is required according to Figures 8 and 10 which show raw data and imaging results of two targets from different azimuth locations by MISO-SAR mode. Figures 9 and 11 show the results by original bidirectional SAR mode with the same parameters.

Furthermore, with the PRF of $5170 \mathrm{~Hz}$, spectra of two targets with the Doppler centroids $-3746 \mathrm{~Hz}$ and $3746 \mathrm{~Hz}$ can be better separated via Doppler band-pass filtering and compressed, but the two spectra cannot be well separated and this phenomenon would introduce high azimuth ambiguities with the PRF of $5490 \mathrm{~Hz}$. The weaker targets are azimuth ambiguities as shown in Figures 8(c) and 

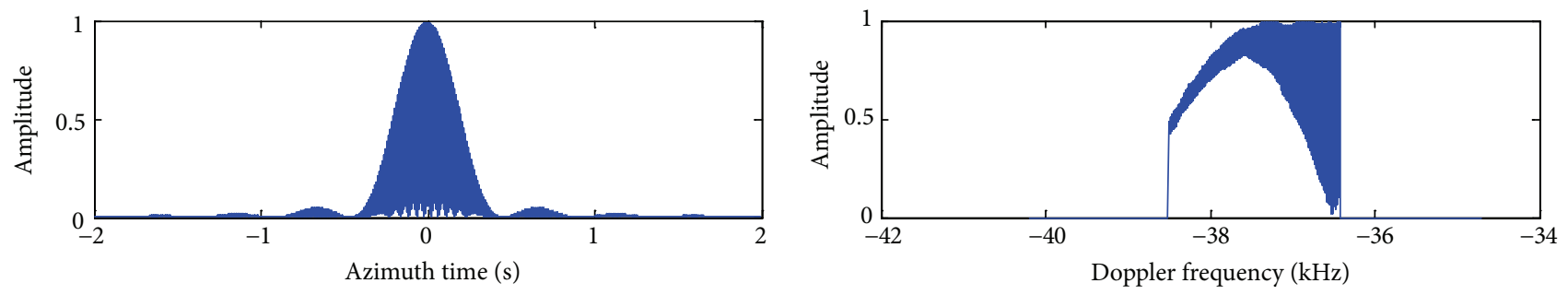

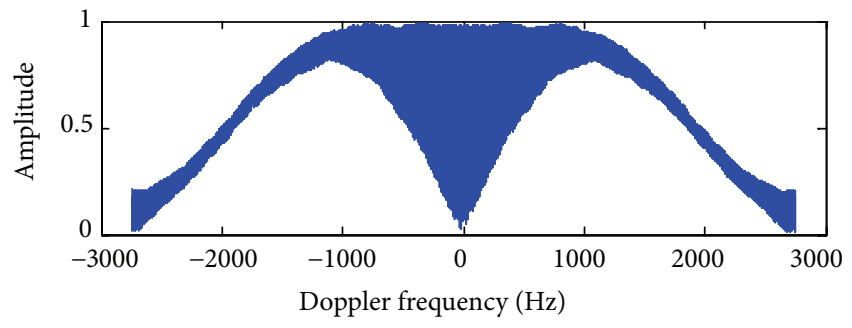

(a)

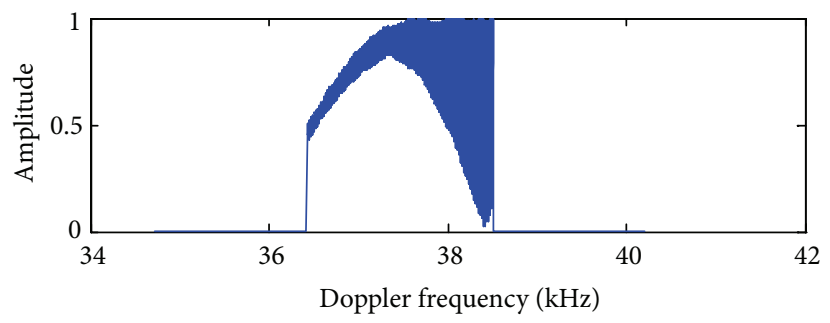

(b)
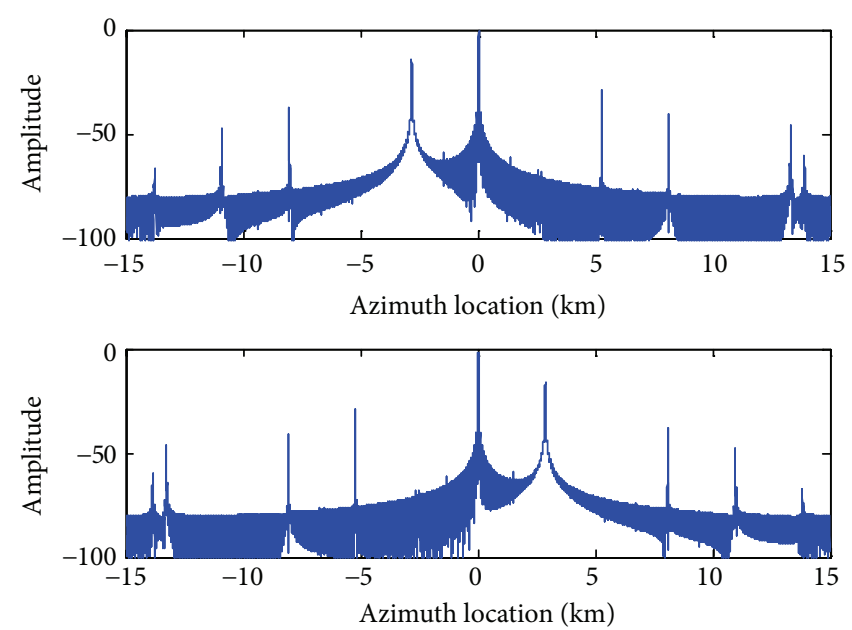

(c)

FIGURE 10: Simulation results of the proposed MISO-SAR with the PRF of $5490 \mathrm{~Hz}$. (a) Azimuth raw data in the time and Doppler frequency domains. (b) Doppler spectra after separation. (c) Imaging results from different azimuth directions.

10(c). Results of Figures 8 and 10 also validate that the AASR oscillates between high and low values versus the $\mathrm{PRF}$ as shown in Figure 4. By comparing with Figures 8 and 9 , it can be seen that the separation and imaging performances by the proposed MISO-SAR are obviously better than those in original bidirectional mode. With the PRF of $5490 \mathrm{~Hz}$, it can lead to the same conclusion by comparing with Figures 10 and 11. Therefore, the superiority of the new MISO-SAR mode has been validated. With the same antenna length and the number of $T / R$ modules, Figure 12 shows simulation results of azimuth raw data and spectra from two azimuth directions in the proposed MIMO-SAR for bidirectional imaging. The operated PRF is $3000 \mathrm{~Hz}$.

\section{Conclusion}

The paper has put forward two novel imaging modes named MISO-SAR and MIMO-SAR for bidirectional imaging, and both modes allow for single-satellite short-term repeated SAR acquisitions in the range of seconds. In the two imaging modes, two subpulses are transmitted to different azimuth locations and the raw data from two directions simultaneously arrive at the sensor and are superimposed into the same receiving window. In the proposed MISO-SAR, echoes from different azimuth directions are separated by Doppler bandpass filtering. Echoes from different azimuth directions in MIMO-SAR are separated in the range-frequency domain due to different carrier frequencies. Compared with the bidirectional imaging mode in [1], the proposed MISO-SAR 

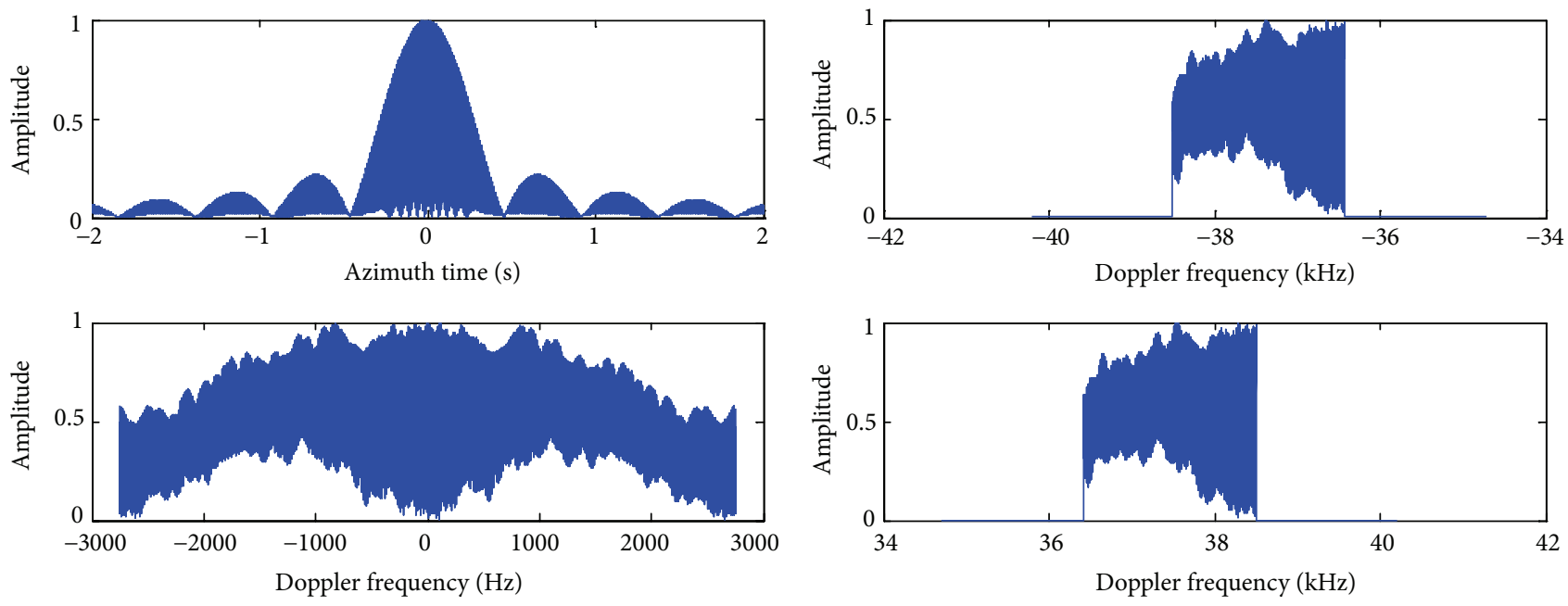

(a)

(b)
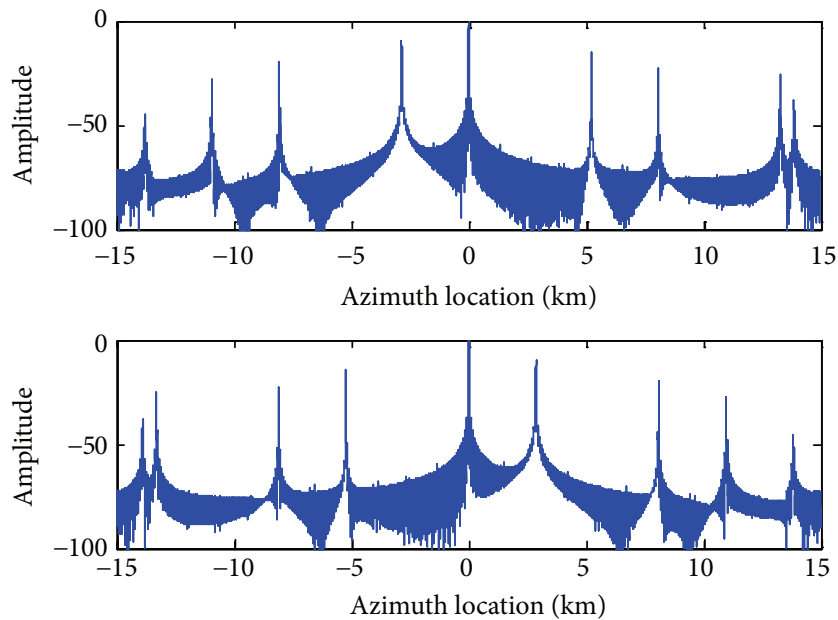

(c)

FIgURE 11: Simulation results of the original bidirectional SAR with the PRF of $5490 \mathrm{~Hz}$. (a) Azimuth raw data in the time and Doppler frequency domains. (b) Doppler spectra after separation. (c) Imaging results from different azimuth directions.
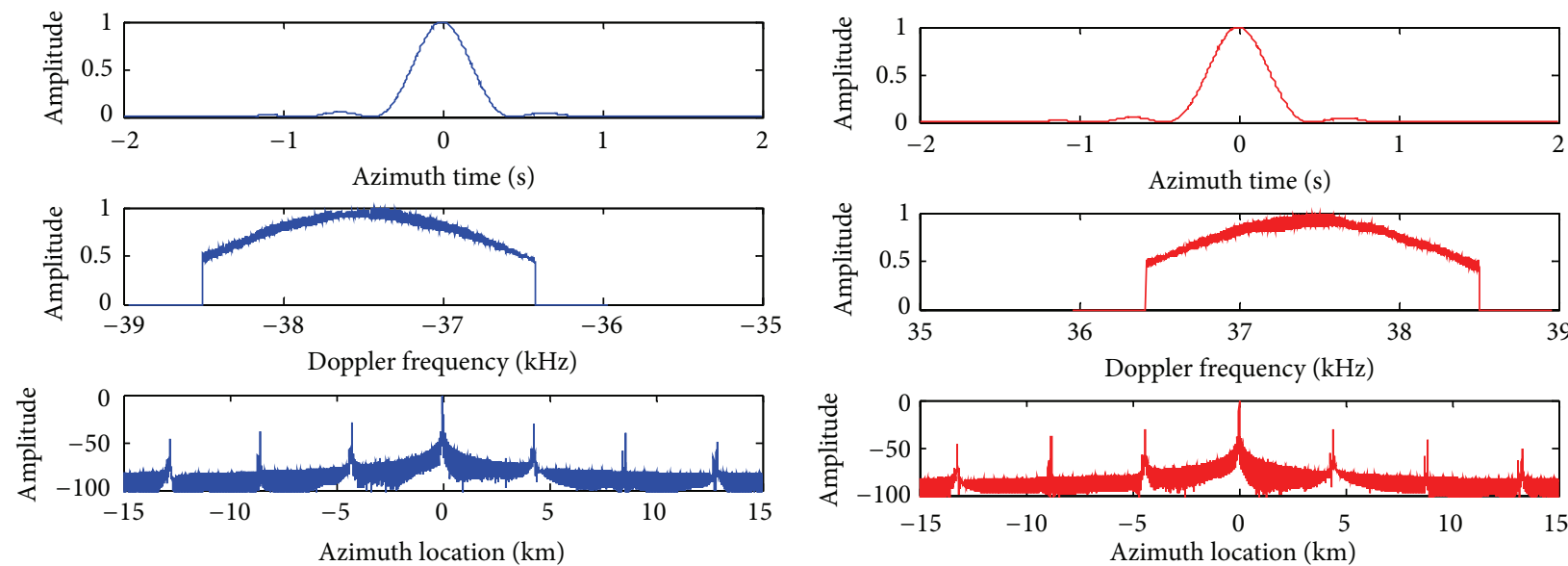

(a)

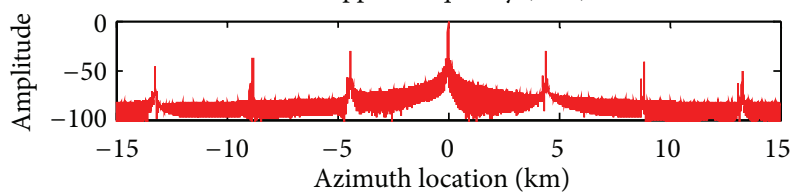

(b)

FIGURE 12: Simulation results of the proposed MIMO-SAR with the PRF of $3000 \mathrm{~Hz}$. (a) Azimuth data of the point target for aft imaging. (b) Azimuth data of the point target for fore imaging. 
and MIMO-SAR modes are with the better AASR under the same condition, especially the proposed MIMO-SAR.

\section{Conflict of Interests}

The authors declare that there is no conflict of interests regarding the publication of this paper.

\section{References}

[1] S. J. Frasier and A. J. Camps, "Dual-beam interferometry for ocean surface current vector mapping," IEEE Transactions on Geoscience and Remote Sensing, vol. 39, no. 2, pp. 401-414, 2001.

[2] D. d'Aria, F. de Zan, D. Giudici, A. M. Guarnieri, and F. Rocca, "Burst-mode SARs for wide-swath surveys," Canadian Journal of Remote Sensing, vol. 33, no. 1, pp. 27-38, 2007.

[3] W. Xu and Y. K. Deng, "Investigation on electronic azimuth beam steering in the spaceborne sar imaging modes," Journal of Electromagnetic Waves and Applications, vol. 25, no. 14-15, pp. 2076-2088, 2011.

[4] S. V. Baumgartner and G. Krieger, "Large along-track baseline SAR-GMTI: first results with the TerraSAR-X/TanDEM-X satellite constellation," in Proceedings of the IEEE International Geoscience and Remote Sensing Symposium (IGARSS '11), S. V. Baumgartner and G. Krieger, Eds., pp. 1319-1322, Vancouver, Canada, July 2011.

[5] J. Mittermayer, S. Wollstadt, S. Baumgartner et al., "Approach to velocity and acceleration measurement in the bi-directional SAR imaging mode," in Proceedings of the IEEE International Geoscience and Remote Sensing Symposium (IGARSS '12), pp. 5618-5621, Munich, Germany, July 2012.

[6] J. Mittermayer, S. Wollstadt, P. Prats-Iraola, P. López-Dekker, G. Krieger, and A. Moreira, "Bidirectional SAR imaging mode," IEEE Transactions on Geoscience and Remote Sensing, vol. 51, no. 1, pp. 601-614, 2013.

[7] J. Mittermayer and S. Wollstadt, "Simultaneous bi-directional SAR acquisition with TerraSAR-X," in Proceedings of the 8th European Conference on Synthetic Aperture Radar (EUSAR '10), J. Mittermayer and S. Wollstadt, Eds., pp. 1-4, Aachen, Germany, 2010.

[8] J. Mittermayer, B. Schattler, and M. Younis, "TerraSAR-X commissioning phase execution summary," IEEE Transactions on Geoscience and Remote Sensing, vol. 48, no. 2, pp. 649-659, 2010.

[9] S. Suchandt, H. Runge, H. Breit, U. Steinbrecher, A. Kotenkov, and U. Balss, "Automatic extraction of traffic flows using TerraSAR-X along-track interferometry," IEEE Transactions on Geoscience and Remote Sensing, vol. 48, no. 2, pp. 807-819, 2010.

[10] J. Mittermayer, S. Wollstadt, P. Prats, P. Lopez-Dekker, G. Krieger, and A. Moreira, "Bi-directional SAR and interferometric SAR short term time series," in Proceedings of the 9th European Conference on Synthetic Aperture Radar (EUSAR '12), pp. 308-311, Nuremberg, Germany, April 2012.

[11] J. H. Gonzalez, M. Bachmann, and H. Hofinann, "TanDEMX commissioning phase status," in Proceedings of the 30th IEEE International Geoscience and Remote Sensing Symposium (IGARSS '10), pp. 2633-2635, Honolulu, Hawaii, USA, July 2010.

[12] J. Mittermayer, M. Younis, R. Metzig, S. Wollstadt, J. M. Martínez, and A. Meta, "TerraSAR-X system performance characterization and verification," IEEE Transactions on Geoscience and Remote Sensing, vol. 48, no. 2, pp. 660-676, 2010.
[13] J. Mittermayer and H. Runge, "Conceptual studies for exploiting the TerraSAR-X dual receive antenna," in Proceedings of the IEEE International Geoscience and Remote Sensing Symposium (IGARSS '03), pp. 2140-2142, Toulouse, France, July 2003.

[14] P. López-Dekker, P. Prats, F. de Zan et al., "Demonstration of SAR interferometry under crossing orbits using TerraSAR$\mathrm{X}$ and TanDEM-X," in Proceedings of the IEEE International Geoscience and Remote Sensing Symposium (IGARSS '11), pp. 3472-3475, Vancouver, Canada, July 2011.

[15] R. Romeiser, S. Suchandt, H. Runge, U. Steinbrecher, and S. Grünler, "First analysis of TerraSAR-X along-track InSARderived current fields," IEEE Transactions on Geoscience and Remote Sensing, vol. 48, no. 2, pp. 820-829, 2010.

[16] R. Scheiber and A. Moreira, "Coregistration of interferometric SAR images using spectral diversity," IEEE Transactions on Geoscience and Remote Sensing, vol. 38, no. 5, pp. 2179-2191, 2000.

[17] G. Krieger, A. Moreira, H. Fiedler et al., "TanDEM-X: a satellite formation for high-resolution SAR interferometry," IEEE Transactions on Geoscience and Remote Sensing, vol. 45, no. 11, pp. 3317-3340, 2007.

[18] R. Bamler and M. Eineder, "Accuracy of differential shift estimation by correlation and split-bandwidth interferometry for wideband and delta-k SAR systems," IEEE Geoscience and Remote Sensing Letters, vol. 2, no. 2, pp. 151-155, 2005.

[19] K. Ouchi, "On the multilook images of moving targets by synthetic aperture radars," IEEE Transactions on Antennas and Propagation, vol. 33, no. 8, pp. 823-827, 1985. 

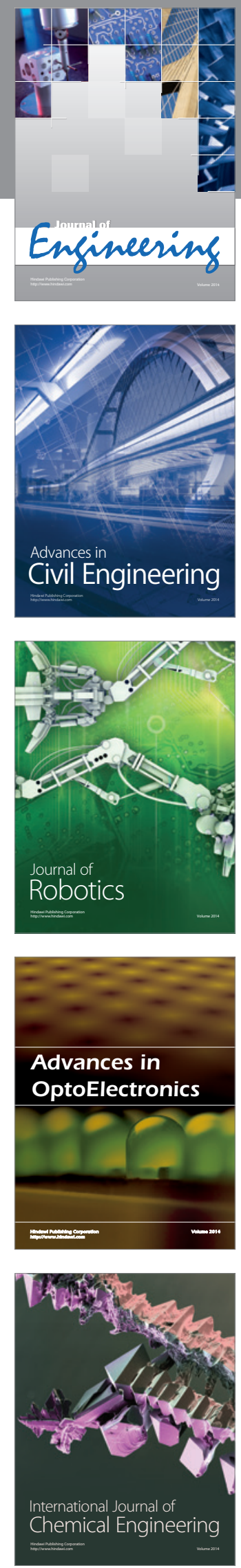

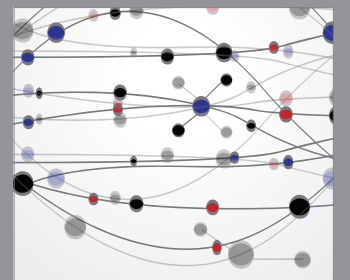

The Scientific World Journal
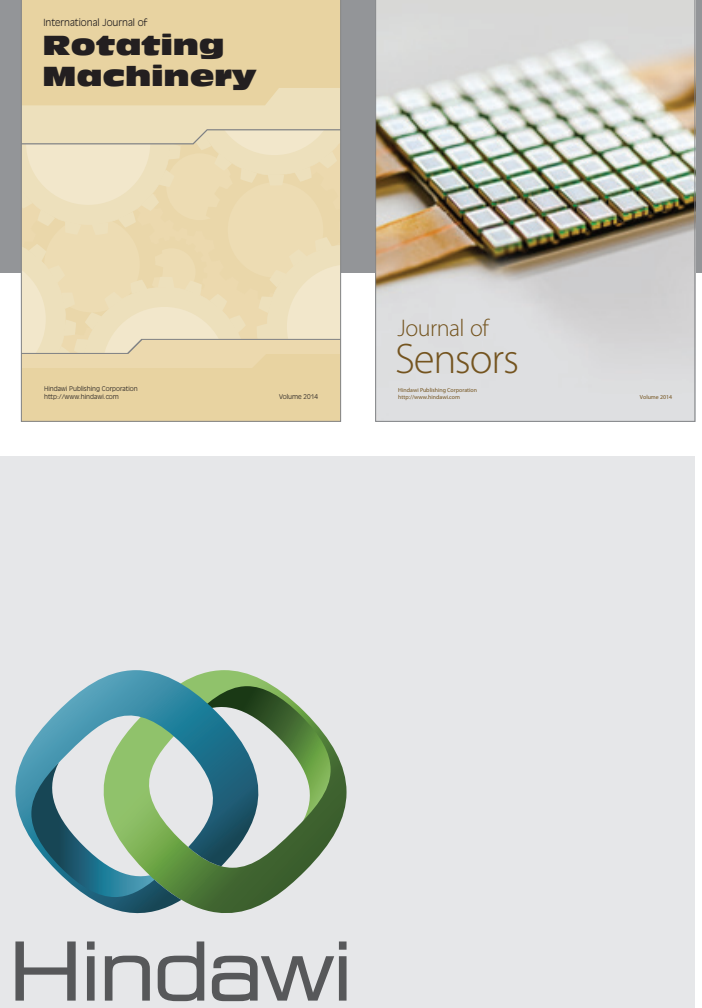

Submit your manuscripts at http://www.hindawi.com
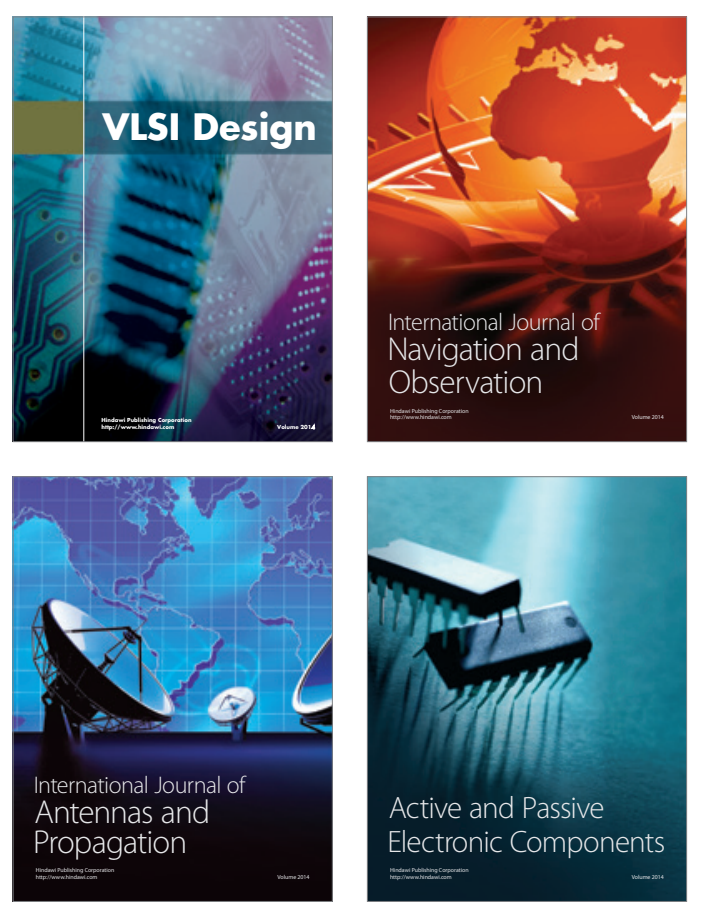
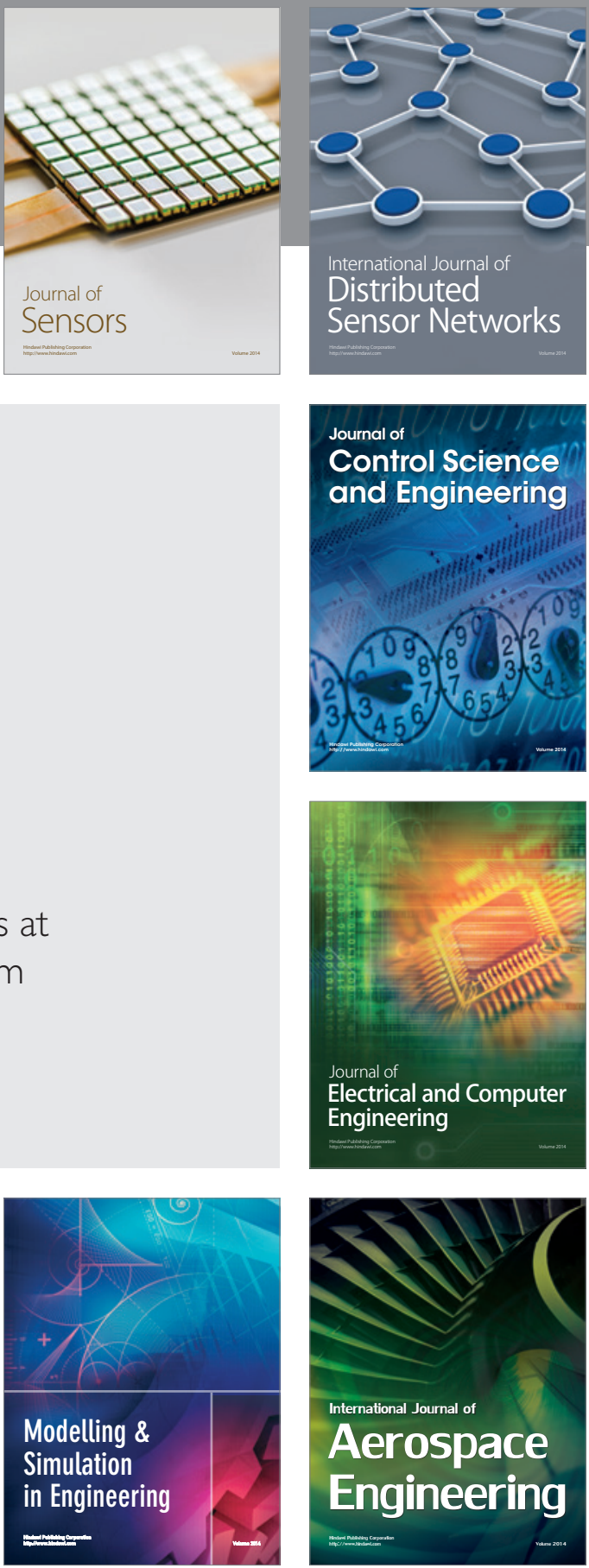

Journal of

Control Science

and Engineering
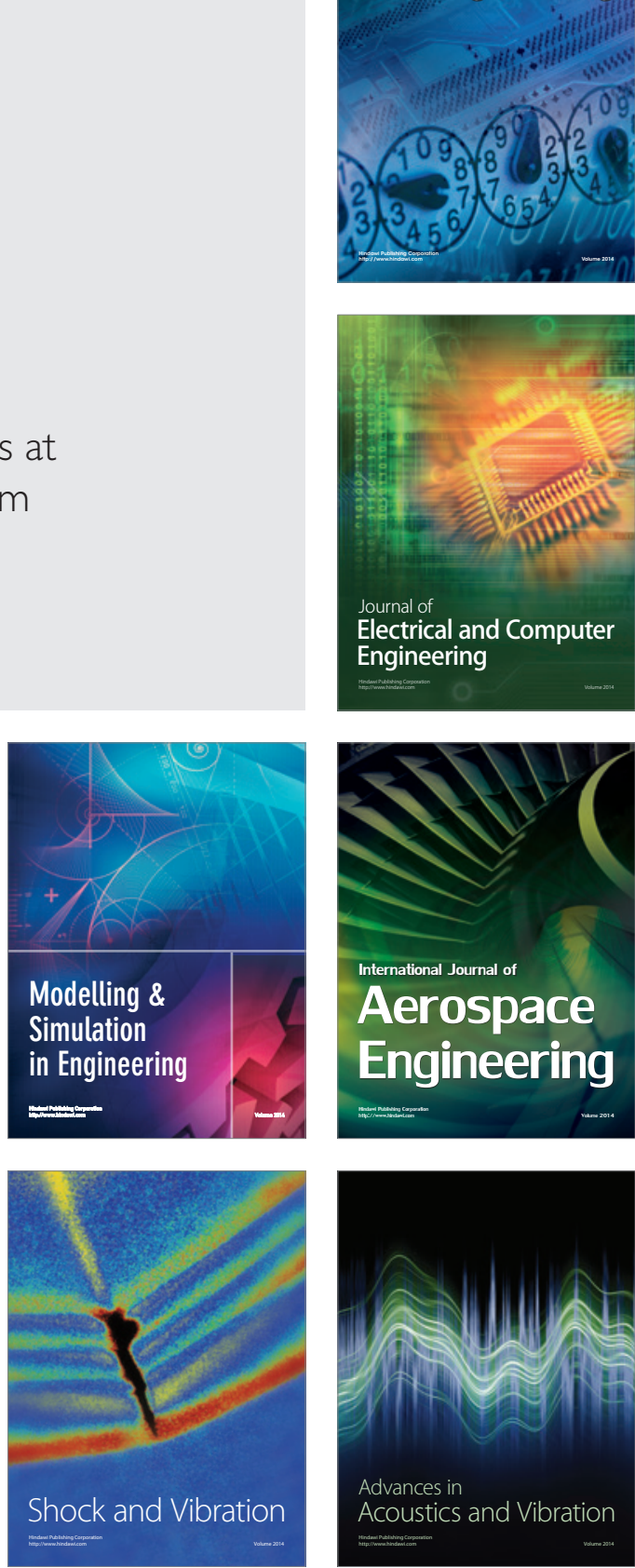